Received: 7 October 2009, Accepted: 7 October 2009

Edited by: M. C. Barbosa

Licence: Creative Commons Attribution 3.0

DOI: 10.4279/PIP.010004

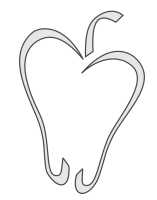

ISSN 1852-4249

\title{
Answer to the Commentary on "A note on the consensus time of mean-field majority-rule dynamics"
}

\author{
Damián H. Zanette ${ }^{1 *}$
}

In his Commentary [1], H. Fort begins by pointing out two aspects of the majority-rule (MR) model that, as presented in the main paper [2], may need some clarification.

It was suggested in the paper that the equivalence of the mean-field MR dynamics and a random walk can be formulated in terms of the evolution of $N_{+}$, the number of agents with opinion +1 . Specifically, in order to write down a master equation for the process, one should find the transition probability that, in a single evolution step, $N_{+}$changes to any other $N_{+}^{\prime}$. It can be readily seen that such event requires that the size $G$ of the group of agents chosen to reach consensus at that step satisfies $G>2|\Delta|$, with $\Delta=N_{+}^{\prime}-N_{+}$. If $\Delta$ is positive (respectively, negative) the number of agents with opinion -1 (respectively, +1 ) in the group must be exactly equal to $|\Delta|$. Summing the probabilities for all the possible values of $G$ yields the transition probability for a given $\Delta$.

Of course, one could cut off and renormalize the probability distribution for the group size, $p_{G}$, in such a way that $G$ can be at most equal to the population size $N$. The two regimes in the size dependence of the consensus time would certainly still exist -although explicitly working out analyt-

\footnotetext{
*E-mail: zanette@cab.cnea.gov.ar

1 Consejo Nacional de Investigaciones Científicas y Técnicas, Centro Atómico Bariloche and Instituto Balseiro, 8400 San Carlos de Bariloche, Río Negro, Argentina.
}

ical results such as Eq. (7) of the main paper may become trickier. However, it is somehow artificial to conceive that, in a social process driven by events which involve agent groups, probabilities depend on the total population size. It sounds more natural to just allow any group size and, in the case that $G \geq N$, admit that the population falls into an absorbing, frozen state. Also, from an operational viewpoint, a cut-off probability distribution with $G \leq N$ would be difficult to implement if the population size varies with time.

The Commentary also addresses a series of generalizations of the MR dynamics -some of which have already been considered in the literaturethat certainly add realism to the model. Heterogeneity among agents is crucial to more realistically approach any population-based complex system. In the context of the MR model, inflexible and unsettled agents as well as "contrarians" have been considered in a series of papers by S. Galam and coworkers (and recently reviewed by Castellano et al. [3]). Also, we have discussed the effects of several forms of heterogeneity on synchronization dynamics [4, 5], which bear close similarities with opinion formation. Overall, the expected consequence of heterogeneity is that full consensus is never reached, but a dynamical state where the degree of consensus fluctuates with time around a well-defined average- ${ }^{-}$is asymptotically approached.

Spatially-distributed populations, where agent groups are localized in space, have been taken into 
Papers in Physics, vol. 1, ART. 010004 (2009) / D. H. Zanette

account since the first formulations of the MR dynamics $[6,7]$. It has been shown that dimensionality has nontrivial consequences in the attainment of consensus. For a fixed group size $G$, the consensus time grows with the population size $N$ as a power whose exponent depends on the dimension [3]. In one-dimensional arrays, the final state is sensibly dependent on the initial condition, while in higher dimensions, the dynamics coincides with diffusive coarsening.

Either random or deterministic time-dependent effects, such as stochastic fluctuations in the rules that govern single consensus events or population dynamics, are certainly worth considering. One may ask, for instance, whether sufficiently frequent "births" or "arrivals" of dissenters are able to overcome consensus attainment. To my knowledge, population dynamics has not been addressed in the context of MR model. On the other hand, randomness in each consensus event has already been taken into account [3].

Finally, global opinion-formation factors such as mass media, advertising, and propaganda play the role of external fields in the spin-like dynamics of the MR model, as also discussed by S. Galam [3].
[1] H Fort, Commentary on "A note on the consensus time of mean-field majority-rule $d y$ namics", Pap. Phys. 1, 010003 (2009).

[2] D H Zanette, A note on the consensus time of mean-field majority-rule dynamics, Pap. Phys. 1, 010002 (2009).

[3] C Castellano, S Fortunato, V Loreto, Statistical physics of social dynamics, Rev. Mod. Phys. 81, 591 (2009).

[4] G H Paissan, D H Zanette, Synchronization of phase oscillators with heterogeneous coupling: A solvable case, Physica D 237, 818 (2008).

[5] D H Zanette, Interplay of noise and coupling in heterogeneous ensembles of phase oscillators, Eur. Phys. J. B 69, 269 (2009).

[6] P L Krapivsky, S Redner, Dynamics of majority rule in two-state interacting spin systems, Phys. Rev. Lett. 90, 238701 (2003).

[7] C J Tessone, R Toral, P Amengual, H S Wio, M San Miguel, Neighborhood models of minority opinion spreading, Eur. Phys. J. B 39, 535 (2004). 